\title{
MANSONELOSIS POR Mansonella ozzardi EN VOLUNTARIOS PARA DESPISTAJE DE MALARIA EN LA AMAZONÍA PERUANA
}

\author{
Javier Vargas ${ }^{1,3, a}$, Nancy Arróspide ${ }^{1, b}$, Sonia Gutiérrez ${ }^{1, b}$, Cristian Obregón ${ }^{1, c}$, Pedro Valencia ${ }^{1,3, d}$, \\ Henry Mormontoy2,b
}

\begin{abstract}
RESUMEN
Objetivos. Determinar la frecuencia, manifestaciones clínicas y factores asociados a la infección por Mansonella ozzardi en voluntarios de una campaña de despistaje de malaria en el distrito de Alto Nanay en la selva amazónica del Perú. Materiales y métodos. Se realizó un estudio descriptivo, analítico y transversal. Los participantes fueron entrevistados y examinados por un médico y los datos fueron registrados en una ficha clínica. El diagnóstico de infección por M. ozzardi se realizó mediante la técnica de gota gruesa y frotis. Resultados. La frecuencia de mansonelosis en esta población fue 47,8\% (IC 95\%: 39,1-56,6). Mediante el análisis bivariado se encontró que el reporte de disminución de la agudeza visual o visión borrosa y la presencia de tumoraciones subcutáneas fueron los signos y síntomas estadísticamente asociados con la infección por microfilarias $(p<0,05)$. La regresión logística encontró asociación estadística para la residencia en localidades del río Pintuyacu o Alto Nanay, la ocupación en lugares alejados del centro poblado, la presencia de tumores subcutáneos y el engrosamiento de la piel $(p<0,05)$. Conclusiones. Existe una elevada frecuencia de mansonelosis por $M$. ozzardi en el distrito de Alto Nanay, la cual se encontró significativamente relacionada con ocupaciones realizadas fuera del centro poblado, residencia en localidades del río Pintuyacu y lesiones cutáneas.
\end{abstract}

Palabras clave: Mansonella ozzardi; Enfermedades desatendidas; Mansonella (fuente: DeCS BIREME).

\section{MANSONELOSIS BY Mansonella ozzardi IN VOLUNTEERS UNDERGOING SCREENING FOR MALARIA IN THE PERUVIAN AMAZON}

\begin{abstract}
Objectives. To determine the frequency, clinical features, and factors associated with $M$. ozzardi infection in volunteers of a malaria screening campaign in the district of Alto Nanay in the Amazon jungle of Peru. Materials and methods. A descriptive, analytical and cross-sectional study was performed. The participants were interviewed and examined by a physician and the data were recorded in a medical record. The diagnosis of M. ozzardi infection was performed using the method of thick blood smear and film. Results. The frequency of mansonelosis in this population was $47.8 \%(95 \%$ $\mathrm{Cl}$ : 39.1 to 56.6). Through bivariate analysis we found that the report of decreased visual acuity or blurred vision and presence of subcutaneous tumors were the signs and symptoms statistically associated with the infection of microfilariae $(p<0.05)$. Logistic regression found statistical association for residency in localities of the Pintuyacu or Alto Nanay rivers, employment in places far from the town center, the presence of subcutaneous tumors and skin thickening $(p<0.05)$. Conclusions. There was a high number of mansonelosis by M. ozzardi in the district of Alto Nanay which was significantly related to working outside the town center, residing in the area of the Pintuyacu River, and skin lesions.
\end{abstract}

Key words: Mansonella ozzardi; Neglected diseases; Mansonella (source: MeSH NLM).

\section{INTRODUCCIÓN}

Las filariasis son parasitosis producidas por nemátodos hemáticos de la familia Filariidae, consideradas dentro del grupo de enfermedades desatendidas (1) con una gran carga de enfermedad (2-4). Mansonella ozzardi, es uno de los agentes etiológicos de mansonelosis distribuido ampliamente en el continente americano y en el Caribe. En América del Sur se han reportado casos en Colombia, Venezuela, Guyana, Surinam, Brasil, Argentina, Perú y Bolivia ${ }^{(5)}$.

En el Perú se describió por primera vez la infección de $M$. ozzardi en cuatro residentes de la ciudad de Iquitos en $1958{ }^{(6)}$. Desde el 2001, Chuquicaña et al. (7), vienen documentando nuevas áreas de propagación

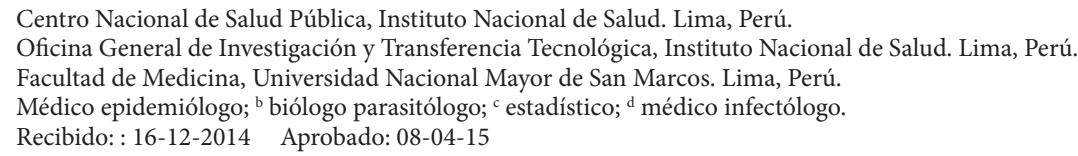


de la infección, tanto en localidades cercanas a lquitos, generalmente en el Bajo Nanay, así como en localidades del río Putumayo y en localidades del río Pintuyacu, en el distrito de Alto Nanay. Usualmente, se reportan casos de mansonelosis en áreas endémicas de malaria, en donde se hallan microfilarias en coinfección con malaria ${ }^{(8,9)}$. En años más recientes, 2009 y 2010, se han venido identificando casos en localidades rurales cercanas a la ciudad de lquitos, refiriendo una procedencia de localidades del distrito de Alto Nanay ${ }^{(10)}$.

La patogenicidad de $M$. ozzardi no es bien conocida. Se le reconocen manifestaciones clínicas inespecíficas que afectan la calidad de vida de los pacientes ${ }^{(5,12)}$. La infección cursa con fiebre, cefalea, mialgias, artralgias, adenopatías, astenia, prurito cutáneo, los cuales también pueden estar presentes en otras enfermedades tropicales prevalentes en la región, como dengue o malaria ${ }^{(11)}$.

El hallazgo de mayor significación en la mansonelosis es la eosinofilia en valores más elevados de lo normal, lo que suele correlacionarse con la densidad parasitaria e intensidad de síntomas pruriginosos (12). Pero se desconocen los factores de riesgo de la infección, los criterios para tratar a una persona residente en una zona de alto riesgo de transmisión, el pronóstico de la enfermedad y sus complicaciones ${ }^{(11)}$.

La incidencia de malaria en el año 2011 fue baja en el Perú y en la región Loreto. El distrito de Alto Nanay, sin embargo, es un área endémica de malaria, en donde siempre se presentan casos, incluso en periodos no epidémicos, por lo que fue seleccionado para buscar activamente casos de la enfermedad, como parte de las actividades de un Programa de evaluación externa del desempeño del diagnóstico de malaria que realiza el Instituto Nacional de Salud. En la localidad de Santa María de Nanay se convocó a residentes que quisieran realizarse pruebas para descartar malaria, encontrándose que muchos estaban infectados con microfilarias de M. ozzardi.

El presente estudio tuvo como objetivo describir la frecuencia, las manifestaciones clínicas y otros factores asociados a la infección por $M$. ozzardien una muestra de la población residente en el distrito de Alto Nanay, conformada por voluntarios de una campaña de despistaje de malaria.

\section{MATERIALES Y MÉTODOS}

\section{DISEÑO Y POBLACIÓN DE ESTUDIO}

Se realizó un estudio descriptivo, analítico y transversal, en participantes voluntarios de una campaña de despistaje de malaria residentes del distrito de Alto
Nanay, con el objetivo de conocer la frecuencia y describir las manifestaciones clínicas y factores asociados a la infección con microfilarias de la especie Mansonella ozzardi mediante la técnica de gota gruesa y frotis.

El estudio se realizó en Santa María de Nanay (101 m de altitud), capital del distrito de Alto Nanay, provincia de Maynas, región Loreto, ubicada a $60 \mathrm{~km}$ al suroeste de Iquitos. El distrito tiene una extensión de 13485,23 km² con 3109 habitantes, según el censo del 2007.

\section{PROCEDIMIENTOS}

En los meses de junio, julio y septiembre de 2011, se realizó una campaña de despistaje a 134 pobladores del distrito de Alto Nanay (por su alto riesgo para la trasmisión de malaria), como parte de los procedimientos para la elaboración de paneles de láminas de gota gruesa y frotis positivas a malaria para el Programa de Evaluación Externa del Desempeño en el Diagnóstico Microscópico de Malaria que ejecuta el INS para los laboratorios de referencia regional del Perú y doce laboratorios de los países de Sudamérica. Los participantes fueron entrevistados y examinados por un médico; los datos proporcionados fueron registrados en una ficha clínica.

El diagnóstico de mansonelosis fue un hallazgo incidental no planificado. Los participantes que presentaron un resultado positivo a Mansonella ozzardi, fueron informados sobre el bajo riesgo de desarrollar una enfermedad grave o discapacitante, $y$, dado que no existe un protocolo de atención establecido para esta enfermedad, a los pacientes que refirieron signos y síntomas se les facilitó ivermectina como tratamiento.

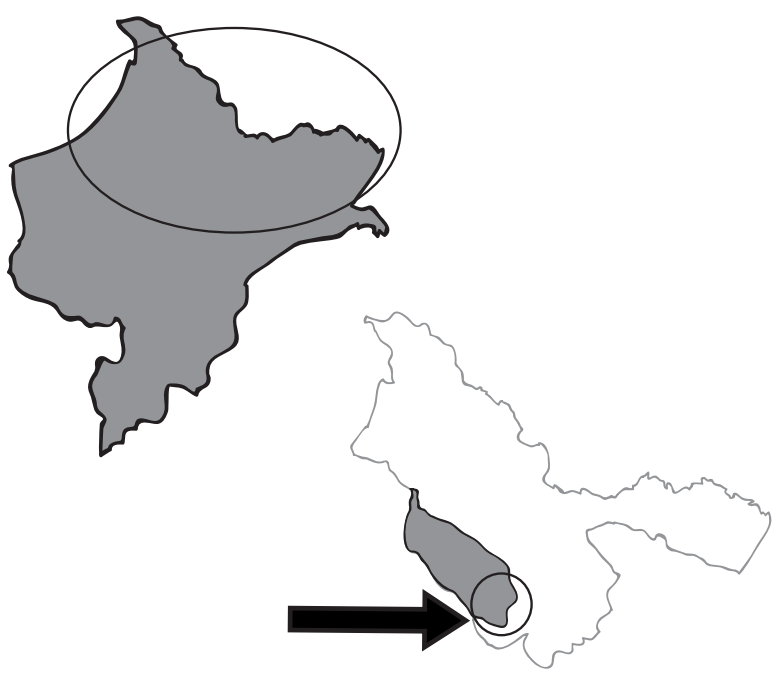

Distrito de Alto Nanay

Figura 1. Ubicación del distrito de Alto Nanay y de la localidad de Santa María en el río Nanay 
La ficha clínica recogió información sobre la fecha de nacimiento, sexo, lugar de nacimiento y de residencia habitual, grado de instrucción, ocupación, historia migratoria y tiempo de residencia. Se solicitó información sobre antecedentes patológicos conocidos de hipertensión arterial, diabetes mellitus u otras enfermedades crónicas, así como diagnóstico de malaria en los últimos 12 meses. La anamnesis registró información sobre erupción dérmica (eritematosa, papular o pruriginosa), edemas, engrosamiento de la piel, agrandamiento de ganglios linfáticos, pérdida de peso, dolores musculoesqueléticos, y disminución de la agudeza visual. El examen físico incluyó la medición del peso, talla, presión sanguínea y presencia de edemas, erupciones dérmicas, agrandamiento de ganglios linfáticos en cuello, axilas e inglés, y nódulos o tumoraciones subcutáneas.

Las filarias fueron identificadas en cuanto a su especie de acuerdo al método de identificación descrito por Thomas C. Orihely y Mark L. Eberhard (13). Este procedimiento fue realizado por dos especialistas, uno hizo la primera lectura en el nivel local para el género y el otro, la segunda lectura en el nivel central para la especie.

\section{ANÁLISIS}

Los datos recolectados fueron ingresados en un formulario electrónico elaborado en el programa Enter y analizado en el programa Analysis del paquete estadístico Epilnfo versión 3.5.1. El análisis estadístico consistió en una descripción de los datos principales y en el análisis bivariado, comparando a las personas infectadas con microfilarias con las personas cuyas muestras de sangre resultaron negativas. Para verificar alguna diferencia entre variables cualitativas de los subgrupos bajo estudio, se utilizó una prueba de chi cuadrado (test exacto de Fisher). Para verificar alguna diferencia entre variables cuantitativas, se utilizó una prueba de $t$ de Student. Las variables que resultaron significativamente asociadas a un resultado positivo a filaria y aquellas con un valor $p<0,1$ en el análisis bivariado, fueron ingresadas a un modelo de regresión logística. Para todas las pruebas estadísticas se eligió como nivel de significancia estadística un valor $p \leq 0,05$.

\section{CONSIDERACIONES ÉTICAS}

Para realizar el procedimiento de toma de muestra de gota gruesa y frotis, los pacientes fueron informados, en forma verbal, del motivo del examen y de las molestias que este les podría causar y solo se realizó el examen de sangre en aquellos que consintieron verbalmente y mediante la firma del consentimiento para la toma de sus muestras hemáticas. En el caso de niños menores de 7 años la decisión de participar fue tomada por los padres; en el caso de los niños de 7 a más años, adicionalmente a la voluntad de sus padres, se le solicitó su asentimiento verbal. Las actividades se realizaron de acuerdo con los procedimientos establecidos en Norma Técnica 054-MINSA/DGSP-V.01 para la atención de malaria y malaria grave, se solicitó opinión al Comité Institucional de Ética en Investigación del Instituto Nacional de Salud para la pertinencia de publicación la cual fue favorable.

\section{RESULTADOS}

La frecuencia de mansonelosis entre 134 pobladores del distrito de Alto Nanay, fue de 47,8\% (IC 95\%; 39,1-56,6). La coinfección de malaria y microfilaria se presentó en tres casos $(2,2 \%)$.

En la Tabla 1 se muestran las características de la infección por M. ozzardi en residentes de Alto Nanay; en la Tabla 2 se muestran los factores que se encontraron significativamente relacionadas con estar infectado por M. ozzardi, entre ellas: la mayor probabilidad en el sexo

Tabla 1. Características de la infección por M. ozzardi en residentes de Alto Nanay, Perú 2011

\begin{tabular}{lcc}
\hline Características generales & $\begin{array}{c}\mathbf{n} \\
\text { positivos }\end{array}$ & $\mathbf{( \% )}$ \\
\hline Sexo & 75 & $(56,0)$ \\
\hline Femenino & 59 & $(44,0)$ \\
\hline Masculino & & \\
Grupos de edad & 2 & $(1,5)$ \\
\hline menores de 5 & 5 & $(3,7)$ \\
\hline 5 a 14 & 25 & $(18,7)$ \\
\hline 15 a 24 & 79 & $(59,0)$ \\
\hline 25 a 59 & 23 & $(17,2)$ \\
\hline 60 a más & & \\
\hline Lugar de residencia & 110 & $(82,1)$ \\
\hline Santa María de Nanay & 16 & $(11,9)$ \\
\hline San Juan de Raya & 4 & $(3,0)$ \\
\hline Saboya & 4 & $(3,0)$ \\
\hline Otros del río Pintuyacu & & \\
\hline Grado de instrucción* & 8 & $(6,6)$ \\
\hline Sin instrucción & 54 & $(44,6)$ \\
\hline Primaria & 33 & $(27,3)$ \\
\hline Secundaria & 26 & $(21,5)$ \\
\hline Superior & & \\
\hline Ocupación & 51 & $(38,1)$ \\
\hline ama de casa & 46 & $(34,3)$ \\
\hline agricultor, pescador, maderero & 15 & $(11,2)$ \\
\hline profesionales, técnicos, empleados & 6 & $(4,5)$ \\
\hline obreros, artesanos & & $(11,9)$ \\
\hline jubilados, estudiantes, dependientes & 16 & \\
\hline
\end{tabular}

* Mayores de 17 años 
Tabla 2. Factores asociados a infección por M. ozzardi en residentes de Alto Nanay, Perú 2011

\begin{tabular}{|c|c|c|c|c|c|}
\hline \multirow{2}{*}{ Factores } & \multicolumn{2}{|c|}{ M. ozzardi } & \multirow{2}{*}{ OR } & \multirow{2}{*}{ (IC 95\%) } & \multirow{2}{*}{ Valor $p$} \\
\hline & Positivo & Negativo & & & \\
\hline \multicolumn{6}{|l|}{ Sexo } \\
\hline Femenino & 28 & 47 & 1,00 & & \\
\hline Masculino & 36 & 23 & 2,63 & $(1,30-5,30)$ & 0,005 \\
\hline \multicolumn{6}{|c|}{ Lugar de residencia } \\
\hline $\begin{array}{l}\text { en la capital del } \\
\text { distrito }\end{array}$ & 44 & 65 & 1,00 & & \\
\hline en caseríos ${ }^{*}$ & 20 & 5 & 5,91 & $(2,06-16,92)$ & $<0,001$ \\
\hline \multicolumn{6}{|c|}{ Grado de instrucción } \\
\hline Superior & 7 & 19 & 1,00 & & \\
\hline $\begin{array}{l}\text { Secundaria o } \\
\text { menos }\end{array}$ & 50 & 45 & 3,01 & $(1,16-7,84)$ & 0,016 \\
\hline \multicolumn{6}{|l|}{ Ocupación } \\
\hline Otra & 57 & 64 & 1,00 & & \\
\hline $\begin{array}{l}\text { Agricultor, } \\
\text { pescador, } \\
\text { maderero }\end{array}$ & 31 & 13 & 4,68 & $(2,09-10,43)$ & $<0,001$ \\
\hline
\end{tabular}

masculino; los residentes en caseríos alejados de Santa María de Nanay; el tener un grado de instrucción de secundaria o menos, quienes tenían como ocupación la agricultura, madereros o pescadores tuvieron dos veces mayor probabilidad.

Los síntomas más frecuentes en las personas infectadas con filariasis fueron: dolores osteomusculares, dolor de cabeza y erupción dérmica. El reporte de disminución de la agudeza visual o visión borrosa se presentó en 5 de 64 afectados $(7,1 \%)$ y fue el único síntoma

Tabla 3. Características clínicas asociadas a infección por M. ozzardi en residentes de Alto Nanay, Perú 2011

\begin{tabular}{|c|c|c|}
\hline Características Clínicas & $n=64$ & $(\%)$ \\
\hline \multicolumn{3}{|l|}{ Síntomas } \\
\hline Dolor osteomuscular & 38 & $(59,4)$ \\
\hline Dolor de cabeza & 26 & $(40,1)$ \\
\hline Erupción dérmica & 25 & $(39,1)$ \\
\hline Hormigueo & 10 & $(15,1)$ \\
\hline Ganglios agrandados & 10 & $(15,1)$ \\
\hline Fiebre & 10 & $(15,1)$ \\
\hline Hormigueo & 10 & $(15,1)$ \\
\hline Baja de peso & 6 & $(9,4)$ \\
\hline Disminución de la agudeza visual* & 5 & $(7,1)$ \\
\hline \multicolumn{3}{|l|}{ Signos } \\
\hline Ganglios linfáticos crecidos & 6 & $(9,4)$ \\
\hline Tumoraciones subcutáneas* & 8 & $(12,5)$ \\
\hline Engrosamiento de la piel & 4 & $(6,3)$ \\
\hline Edema en miembros inferiores & 1 & $(0,7)$ \\
\hline
\end{tabular}

${ }^{*} p<0,05$
Tabla 4. Factores asociados a infección por M. ozzardi en residentes de Alto Nanay, Perú 2011

\begin{tabular}{lccc}
\hline \multicolumn{1}{c}{ Variable } & OR & (IC 95\%) & Valor $\boldsymbol{p}$ \\
\hline Residencia & 5,38 & $(1,76-16,47)$ & 0,003 \\
Ocupación & 4,39 & $(1,89-10,19)$ & 0,001 \\
Tumoración subcutánea & 7,71 & $(1,46-40,74)$ & 0,016 \\
Engrosamiento de piel & 9,89 & $(1,01-96,51)$ & 0,048 \\
\hline
\end{tabular}

significativamente relacionado con la infección por M. ozzardi (Tabla 3).

Los signos más fencontrados en los afectados fueron ganglios linfáticos palpables y tumoraciones subcutáneas, como lipomas, localizadas en cuero cabelludo, espalda y miembros superiores e inferiores. Este último hallazgo estuvo estadísticamente asociado con la infección por microfilarias $(p=0,03)$ (Tabla 3).

En el análisis multivariado de regresión logística los factores asociados a infección por $M$. ozzardi fueron residencia (en localidades del río Pintuyacu o Alto Nanay); ocupación (en lugares alejados del centro poblado); la presencia de tumores subcutáneos y el engrosamiento de la piel. Este modelo explica el 97,2\% de los casos de infección por microfilarias (Tabla 4).

\section{DISCUSIÓN}

La infección por filarias de $M$. ozzardi es una enfermedad olvidada y completamente desatendida ${ }^{(1)}$, tanto por el Estado como por la sociedad civil de la zona geográfica en donde se presenta. Afecta a las poblaciones rurales más pobres y con menos accesibilidad a los servicios de salud de todos los territorios de la cuenca amazónica de los países sudamericanos ${ }^{(14-17)}$ entre los que se destaca Brasil, por haber aportado mayor información acerca de su extensión en su vasto territorio y de las características clínicas y epidemiológicas.

El presente estudio revela que la infección por M. ozzardi es un problema de gran magnitud en la cuenca del río Nanay, su frecuencia en participantes voluntarios de una campaña de salud, residentes en el distrito de Alto Nanay alcanza el $47,8 \%$ y, si bien fue significativamente mayor en varones, trabajadores del campo, del río o del bosque, y residentes de zonas más rurales; se encuentran casos en todos los grupos de población. Este resultado es consistente con el reporte realizado por Jones et al. (18) quienes reportaron una frecuencia de $49 \%$ para la localidad de Santa María de Nanay. Así mismo, estudios de prevalencia con muestras aleatorias, realizados en el Brasil en poblaciones amazónicas 
similares, han documentado prevalencias de infección por $M$. ozzardi mayores a $10 \%$ en Coari ${ }^{(19)}$, mayores a $20 \%$ en Purus ${ }^{(5)}$ o mayores $30 \%$ en Labrea ${ }^{(14)} \mathrm{y}$, de igual manera, encontraron prevalencias mayores en varones que realizan ocupaciones fuera de los centros poblados así como en residentes de áreas más rurales.

La infección con microfilarias de $M$. ozzardi ya se había documentado en poblados ubicados cerca de la desembocadura del río Nanay en el río Amazonas ${ }^{(20)}$, en la ciudad de lquitos. Al parecer, su frecuencia se va incrementando en las poblaciones río arriba, hacia la cabecera del río Nanay y de sus afluentes, los ríos Pintuyacu y Chambira. En este estudio se ha observado que la frecuencia fue significativamente mayor en los residentes de los caseríos ubicados en el río Pintuyacu, donde alcanzó hasta $87,5 \%$ en personas procedentes de la comunidad San Juan de Raya. La mayor frecuencia en áreas más rurales probablemente tenga relación con la mayor densidad de los vectores trasmisores de $M$. ozzardi, pero está fuera del alcance de este estudio afirmarlo. En Coari, en el estado de Amazonas, la región más cercana a la región Loreto en el Perú, Martins et al. (19) encontraron que 5,6\% de Simuliidae de la especie Cerqueirellum argentiscutum, capturados en la zona urbana estaban infectados con M. ozzardi, mientras que $7,1 \%$ de mosquitos capturados en la zona rural también lo estaban.

En áreas endémicas de malaria, la coinfección de malaria y filaria es muy frecuente y su hallazgo incidental es reportado por los microscopistas que leen láminas de gota gruesa, y que conocen también las microfilarias y las encuentran en muestras en las que buscan plasmodios, además, existen una serie de reportes de su hallazgo incidental. El distrito de Alto Nanay es un área endémica de malaria; en el año 2013 tuvo un índice palúdico anual de 565 por mil y fue el segundo distrito con mayor incidencia de malaria a nivel nacional (21). Cuando se realizó este estudio, en el año 2011, su frecuencia fue baja, pese a ello, la frecuencia de malaria fue de $3,7 \%$ y la coinfección de plasmodios con microfilarias fue de $2,2 \%$.

En general, los textos de Parasitología y Medicina consideran que la filariasis por $M$. ozzardi es asintomática o presenta signos y síntomas inespecíficos (22-24), pero Batista et al. (12) reportaron ya en 1960, que esta se acompaña de dolores articulares, frialdad en las piernas, adenitis inguinocrurales, placas eritematosas y pruriginosas, y dolor de cabeza, los cuales fueron significativamente mayores entre los pacientes infectados que en las personas libres de la infección, y que la intensidad de los síntomas se relaciona con una mayor densidad parasitaria. Más recientemente, Martins et al. ${ }^{(19)}$, corroboraron la presencia de un cuadro clínico similar en Coarí. En un reporte, que da cuenta de una evaluación más específica de cuatro casos de filariosis por M. ozzardi, se verificó también que los pacientes con mayores densidades parasitarias presentaban una intensidad mayor de los síntomas ${ }^{(11)}$.

En el presente estudio, los pacientes han reportado con más frecuencia dolor osteomuscular, dolor de cabeza y erupción dérmica. El examen físico reveló tumoraciones subcutáneas, adenopatías y engrosamiento de la piel, pero en el análisis bivariado, solo se encontró asociación significativa con la infección por $M$. ozzardi, al reporte de disminución de la agudeza visual y a la presencia de tumoraciones subcutáneas. Posteriormente, un análisis multivariado de los factores que estuvieron significativamente relacionados con la infección por microfilarias, desestimó el reporte de la baja de la agudeza visual y mostró que el perfil de un residente del distrito de Alto Nanay infectado corresponde a una persona que reside en zona más rural, cuya ocupación se realiza fuera del centro poblado y que presenta engrosamiento de la piel y tumoraciones subcutáneas.

Este último hallazgo corresponde a una lesión parecida a un lipoma que se ubica frecuentemente en cuero cabelludo, espalda y miembros superiores e inferiores. Al describir su ciclo vital, se cita que los adultos se alojan en los tejidos grasos de las cavidades corporales, pero también en el tejido subcutáneo. Estas tumoraciones subcutáneas, conocidas en la población como "lobanillos", no se describen como relacionadas con M. ozzardi, por lo que parece relevante investigarlas más profundamente.

Recientemente Arróspide et al. ${ }^{(10,25)}$ notificaron en muestras de sangre de personas residentes en comunidades del río Nanay, la existencia de una variedad de Mansonella a la que se denominó atípica debido a la disposición ligeramente diferente de los primeros núcleos detrás del espacio cefálico. Estudios filogenéticos moleculares establecieron que se trataba de especies de $M$. ozzardi que estarían atravesando un proceso de la variación natural de la especie. En el presente estudio se encontraron estas mansonelas atípicas en tres participantes. Es posible pensar que las variaciones naturales que atraviesa el parásito también se relacionen con variaciones en su ciclo vital $\mathrm{y}$, consecuentemente, nuevas características patogénicas.

La principal limitación del presente estudio es que no utilizó una muestra aleatoria de la población del distrito de Alto Nanay para medir la prevalencia de la infección por microfilarias, porque no fue un estudio 
planificado sino un hallazgo relevante. Las personas que voluntariamente participaron en una campaña de salud, pueden estar motivadas por atender alguna molestia y esta podría estar relacionada con la infección por microfilarias, por lo que la frecuencia de infección por M. ozzardi en esta población, puede ser más elevada que la real. Aun así, se puede establecer que la frecuencia de infección por este parásito en la población de voluntarios a despistaje de malaria en el distrito de Alto Nanay es alta: $47,8 \%$. Esta frecuencia hallada concuerda con la reportada por Jones et al. (18) en la misma localidad; probablemente en las comunidades más rurales y alejadas de Santa María la frecuencia sea mayor. Otras limitaciones fueron la falta de estudio de las tumoraciones subcutáneas y de otros exámenes oftalmológicos y de laboratorio especializados, que hubieran permitido proporcionar más evidencia de la relación entre la infección y las lesiones encontradas; por ejemplo, establecer si el reporte de disminución de la agudeza visual de los pacientes se relaciona con lesiones corneales que se han encontrado en pacientes infectados con M. ozzardi en otro estudio ${ }^{(26)}$
El presente estudio es relevante porque logra identificar factores que incrementan la probabilidad de contraer la mansonelosis en comunidades ribereñas de la Amazonía peruana, su magnitud y características clínicas. Es un avance en el esfuerzo por conocer aspectos más específicos que permitan establecer protocolos, caracterizar el cuadro clínico, recomendar el método diagnóstico más apropiado en la zona rural, recomendar cuándo decidir tratar a una persona infectada, qué medicamento utilizar y por cuánto tiempo, lo cual podría ser resuelto en estudios de cohortes de personas jóvenes libres de la infección.

Contribuciones de autoría: JV, NA y SG diseñaron el estudio, JV y SG tomaron las muestras y evaluaron a los participantes. NAidentificó las especies de las filarias halladas, CO dio asesoría estadística. JV, NA, HM participaron en la redacción del artículo. JV, NA, CO, HM criticaron y aprobaron la versión final a publicar, PV participo en la asesoría técnica y administrativa.

Agradecimientos: al personal de salud y a la población del distrito de Alto Nanay. A Franklin Chirinos Palomino y Melitón Gebol Cahuaza

Conflictos de interés: los autores declaran no tener conflictos de interés.

\section{REFERENCIAS BIBLIOGRÁFICAS}

1. World Health Organization. Lynphatic Filariasis. Progress report 2000-2009 and strategic plan 20102020 of the global programme to eliminate lymphatic filariasis: halfway towards eliminating lymphatic filariasis. Geneva: WHO; 2010.

2. Mathers CD, Ezzati M, Lopez AD. Measuring the burden of neglected tropical diseases: the global burden of disease framework. PLoS Negl Trop Dis. 2007 Nov 7;1(2):e114.

3. Basañez MG, Pion SD, Churcher TS, Breitling LP, Little MP, Boussinesq M. River blindness: a success story under threat? PLoS Med. 2006 Sep;3(9):e371.

4. Wynd S, Melrose WD, Durrheim $\mathrm{DN}$, Carron J, Gyapong $\mathrm{M}$. Understanding the community impact of lymphatic filariasis: a review of the sociocultural literature. Bull World Health Organ. 2007 Jun;85(6):493-8.

5. Medeiros JF, Py-Daniel V, Barbosa UC, Izzo TJ. Mansonella ozzardi in Brazil: prevalence of infection in riverine communities in the Purus region, in the state of Amazonas. Mem Inst Oswaldo Cruz. 2009 Feb;104(1):74-80.

6. Gonzales-Mugaburu L. Hallazgo de Mansonella ozzardi en la selva peruana: nota preliminar. Rev Peru Med Exp Salud Publica. 1958;12(1-2):87-9.

7. Chuquicaña A, Durand S, Bentley G, Sanchez JF, Yalta A, Zerpa R, et al. Prevalencia y distribución de filariasis por Mansonella ozzardi en la Cuenca Amazónica Peruana. I Conferencia anual de la Sociedad Norteamericana de Medicina tropical e Higiene en el Perú. Lima, Perú. Febrero de 2011.

8. Vargas J, Arróspide N, Gutierrez S, Chirinos F, Gebol M. Filariosis por Mansonella ozzardi en la región Loreto, una infección desatendida. Bol Inst Nac Salud. 2012;18(5-6):93-5.

9. Dantur Juri MJ, Veggiani Aybar CA, Ortega ES, Galante GB, Zaidenberg MO. Plasmodium vivax and Mansonella ozzardi co-infection in north-western Argentina. Malar J. 2013 Jul 17;12:248. doi: 10.1186/1475-2875-12-248.

10. Arróspide N, Adami YL, Gutiérrez S, Vargas J. Morphological characterization of atypical and Ozzardi microfilariae of mansonella gender. Rev Peru Med Exp Salud Publica. 2012 Mar;29(1):161-3.

11. Vargas-Herrera J, Arróspide-Velasco N, Gutierrez-González S, CelisSalinas JC, Huamaní-Solano D, Loza-Hermenegildo L, et al. Report of four clinical cases of filariasis in Alto Nanay, Loreto. Rev Peru Med Exp Salud Publica. 2013 Jul;30(3):506-11.

12. Batista D, Oliveira WR, Rabello VD. Estudo da patogenicidade da Mansonella ozzardi e da sintomatologia da mansonelose. Rev Inst Med Trop São Paulo. 1960;2:281-9.

13. Orihel TC, Eberhard ML. Mansonella ozzardi: a redescription with comments on its taxonomic 
relationships. Am J Trop Med Hyg. 1982 Nov;31(6):1142-7.

14. Medeiros JF, Py-Daniel V, Barbosa UC, Ogawa GM. Current profile of Mansonella ozzardi (Nematoda: Onchocercidae) in communities along the Ituxi river, Lábrea municipality, Amazonas, Brazil. Mem Inst Oswaldo Cruz. 2008 Jun;103(4):409-11.

15. Lawrence DN, Erdtmann B, Peet JW, Nunes de Mello JA, Healy GR, Neel JV, et al. Epidemiologic studies among Amerindian populations of Amazonia. II. Prevalence of Mansonella ozzardi. Am J Trop Med Hyg. 1979 Nov;28(6):991-6.

16. Bartoloni A, Cancrini G, Bartalesi F, Marcolin D, Roselli M, Arce CC, et al. Mansonella ozzardi infection in Bolivia: prevalence and clinical associations in the Chaco region. Am J Trop Med Hyg. 1999 Nov;61(5):830-3.

17. Medrano CE, Volcán GS, Godoy GA. [Mansonelliasis in the southeast Venezuelan Orinoquia region]. Rev Inst Med Trop Sao Paulo. 1992 Jan-Feb;34(1):61-70. [Article in Spanish]

18. Jones E, Durand S, Bentley G, Nattell N, Tapia L. Rimarachin D. Braga G. Chuquicana A. Baldeviano G. Edgel K. Lescano A.G. Variable prevalence but limited clinical features of filariasis by Mansonella ozzardi in the peruvian amazon basin.
Journal of Investigative Medicine. 2012;60(1):445.

19. Martins M, Pessoa FA, de Medeiros MB, de Andrade EV, Medeiros JF. Mansonella ozzardi in Amazonas, Brazil: prevalence and distribution in the municipality of Coari, in the middle Solimões River. Mem Inst Oswaldo Cruz. 2010 May;105(3):246-53.

20. Chuquicaña A, Durand S, Bentley G, Sánchez JF, Yalta Flores A, Zerpa $\mathrm{R}$, et al. Prevalencia y distribución de filariasis por Mansonella ozzardi en la Cuenca Amazónica Peruana [Internet]. I Conferencia Anual de la Sociedad Norteamericana de Medicina Tropical e Higiene en el Perú. Lima: Sociedad Norteamericana de Medicina Tropical e Higiene; Febrero de 2011 [citado el 13 de abril de 2014]. Disponible en: http://www. epiredperu.net/epired/eventos/ W E B \% 20 I \% 20 A S T M H \% 20 Peru\%202011/ Poster\%20 presentation $/ 29$ - Poster $\% 20$ -\%20 Chuquica \% C $3 \%$ B 1 a $\% 20$ - \% 20 Prevalencia $\% 20$ y $\% 20$ distribucion $\% 20$ de $\% 20$ filiariasis. pdf

21. Dirección General de Epidemiología. Resumen de las enfermedades o eventos bajo vigilancia epidemiológica en el Perú, del 08 al 14 de Diciembre de 2013. Bol Epidemiol (Lima). 2013; 22 (50): 1044-8.
22. Botero D, Restrepo M. Filariosis. En: Botero D, Restrepo M. Parasitosis Humanas. Capítulo 11. 3ra edición. Medellín: Editorial Corporación para investigaciones biológicas; 1998.

23. Duménigo Ripoll B. Filariasis. En: Llop A, Valdés-Dapena M, Zuazo J. Microbiología y Parasitología Médicas. Capítulo 101. 1ra Edición. La Habana: Editorial Ciencias Médicas; 2001.

24. Pezzani, Betina C. Filarias. En: Basualdo JE, Coto CE, De Torres RA. Microbiología Biomédica. Capítulo 142. Buenos Aires: Editorial Atlante; 2006.

25. Adami YL, Moraes MA, Lanfredi RM, Maia-Herzog M. An atypical microfilaria in blood samples from inhabitants of Brazilian Amazon. Parasitol Res. 2008 Dec;104(1):959. doi: 10.1007/s00436-008-1164-4.

26. Vianna LM, Martins M, Cohen MJ, Cohen J.M. Belfort Jr. Mansonella ozzardi corneal lesions in the Amazon: A cross-sectional study. BMJ Open. 2012 Nov 27;2(6). pii: e001266. doi: 10.1136/ bmjopen-2012-001266.

Correspondencia: Nancy Arróspide

Dirección: Capac Yupanqui 1400

Teléfono 51-1-7481111 anexo 2168

Correo electrónico:narrospide@ins.gob.pe

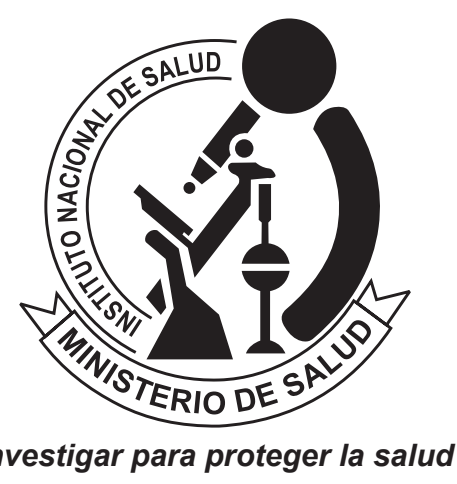

REVISTA PERUANA DE MEDICINA EXPERIMENTAL Y SALUD PÚBLICA CUMPLIENDO SUS METAS Y PROYECTÁNDOSE AL FUTURO

\section{Visite los contenidos de la revista en: www.ins.gob.pe/rpmesp}

